\title{
ALGEBRAIC APPROACH USING STRUCTURED SINGULAR VALUE: APPLICATION TO A NONLINEAR PLANT
}

\author{
Marek Dlapa \\ Department of Automation and Control Engineering \\ Faculty of Applied Informatics \\ Tomas Bata University in Zlin \\ nám. T. G. Masaryka 5555, 76001 Zlín, Czech Republic \\ E-mail: dlapa@fai.utb.cz
}

\section{KEYWORDS}

Algebraic approach, controller design, PID control, structured singular value, Differential Migration, evolutionary algorithms.

\begin{abstract}
The contribution describes an application of the algebraic $\mu$-synthesis methodology to the control of a real plant with a nonlinear characteristic. The controller design is considered as a problem of minimization of the peak of the structured singular value denoted $\mu$. The algebraic approach consists of the pole placement principle based on the polynomial Diophantine equations and Differential Migration procedure used for optimization. The results are compared with other controllers designed via the $D-K$ iteration, synthesis in the ring of proper and stable functions and the Naslin method.
\end{abstract}

\section{INTRODUCTION}

Intensive research activity performed in the robust control theory during the recent years has brought new methods considering both the parametric and dynamic structured uncertainties. Some of the methods are based on the $\mathbf{H}_{\infty}$ approach in the ring of stable and proper transfer functions denoted $R_{\mathrm{PS}}$. These methods provide a measure that indicates the robustness of designed controller. However, this measure evaluates only the robust stability. On the other hand, methods based on the Zames' small gain theorem (Zames 1981) yield both the robust stability and performance conditions. One of them is the structured singular value denoted $\mu$ (Doyle 1982) which treats the robust stability and performance objectives simultaneously. Two methods for the $\mu$-synthesis were derived: the $D-K$ iteration (Doyle 1985) and $\mu-K$ iteration (Lin et al. 1993). The $D-K$ iteration yields a suboptimal controller minimizing the peak of the upper bound for $\mu$-function. However, the controller has usually a high order transfer function due to the scaling matrices $D, D^{-1}$ and for further application it is simplified via some kind of approximation. If the simplification is too substantial it can cause degradation of the frequency properties of the controller and the whole feedback loop. In some cases, the scaling matrices cannot be approximated with the desired precision and the resulting controller can be far from the optimality. Moreover, the state space formulae for the $\mathbf{H}_{\infty}$ suboptimal controller require the stability of the performance weighting function (Doyle et al. 1989). These problems can be resolved using the algebraic $\mu$-synthesis (Dlapa et al. 2009, Dlapa and Prokop 2010) treated in this contribution, which overcomes both the approximation of the scaling matrices $D, D^{-1}$ and the impossibility of integrating behaviour of the performance weighting function. In this method the controller is designed through the algebraic pole placement principle applied to the nominal plant and the position of the nominal closed-loop poles is tuned through an evolutionary algorithm with evaluation of the upper bound for $\mu$. The problem of instability of performance weighting function is utilized by setting the nominal closed-loop poles to the real axis in the left half-plane.

In this paper, the algebraic $\mu$-synthesis and $D-K$ iteration are applied to a plant with nonlinear behaviour and the resulting controllers are compared with other standard methods.

\section{PLANT DESCRIPTION AND IDENTIFICATION}

The control of heating systems has been an important field in the control theory for decades. There is a number of applications of temperature control involving nonlinear and time-delay systems present in the electrical and heating industry as well as in technology processes (e.g. Fiser 2002 or Liu 2003).

The problem of nonlinear control can be utilized by an adaptation to parameters changes or by using a robust or nonlinear controller. The usage of the adaptive control is limited by recursive identification, which has not satisfactory results when the input to the controller is subject to noise or if there are other factors, such as timedelay or external disturbances causing inaccuracy of measured signals. The algebraic $\mu$-synthesis treated in this contribution is more versatile than common methods for the robust control and can consider the effects of noise, nonlinearity and time-delay as well as the influence of external disturbances. However, the usage of this method is limited to the models represented by linear fractional transformation (LFT). In order to describe nonlinearity of controlled plant the parametric uncertainty is used and transformed to the LFT framework which does not increase conservatism of the plant model.

The air-heating set considered in this contribution has three input and seven measured quantities. The input signals are the voltage on bulb and the main and 
adjacent fan. The circuit was controlled by a standard IBM PC computer, which communicates via serial link (RS232) with the CTRL unit. The CTRL unit converts the digital data to unified analogue signals. In the transformation and unification unit the unified analogue signals are transformed to the voltage on a particular actuator. Similarly, the measured signals are transformed to the unified voltage $0-10 \mathrm{~V}$.

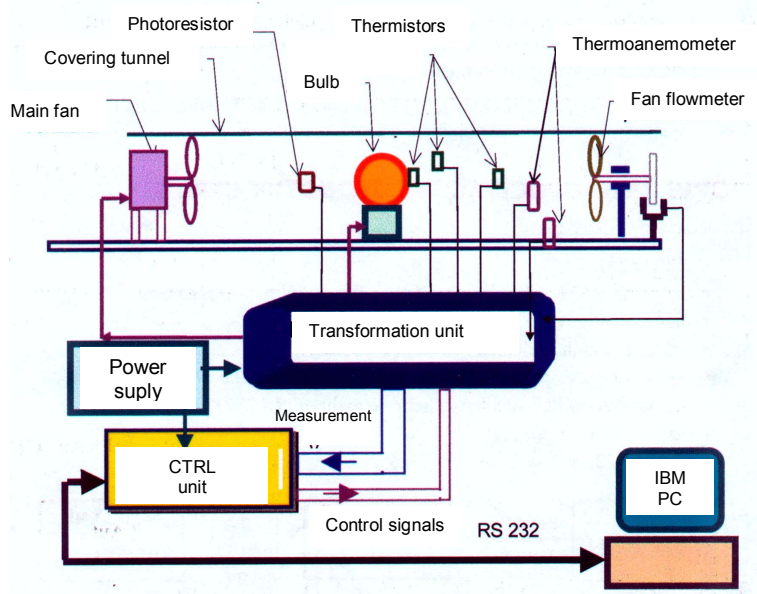

Figure 1: Plant Scheme

Table 1: Input and Output Channels of CTRL Unit

\begin{tabular}{|c|l|c|l|}
\hline $\begin{array}{c}\text { Input } \\
\mathrm{s}\end{array}$ & \multicolumn{1}{|c|}{ Sensor } & Outputs & \multicolumn{1}{|c|}{ Actuator } \\
\hline$y 1$ & sensor of bulb radiance & $u 1$ & voltage of bulb \\
\hline$y 2$ & $\begin{array}{l}\text { sensor of temperature near } \\
\text { bulb } \mathrm{T}_{2}\end{array}$ & $u 2$ & $\begin{array}{l}\text { voltage of main fan } \\
\text { (speed control) }\end{array}$ \\
\hline$y 3$ & $\begin{array}{l}\text { temperature of envelope of } \\
\text { the bulb T }\end{array}$ & $u 3$ & $\begin{array}{l}\text { voltage of adjacent fan } \\
\text { (speed control) }\end{array}$ \\
\hline$y 4$ & $\begin{array}{l}\text { temperature at output of } \\
\text { tunnel } \mathrm{T}_{4}\end{array}$ & & \\
\hline$y 6$ & termoanemometer $\mathrm{TA}_{6}$ & & \\
\hline$y 7$ & fan flowmeter & & \\
\hline
\end{tabular}

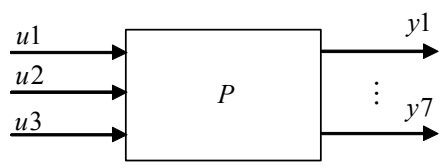

Figure 2: Inputs and Outputs of Plant

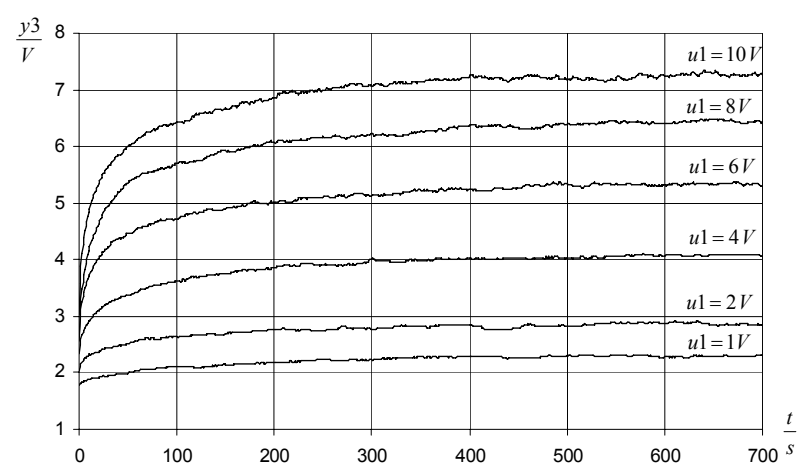

Figure 3: Step Responses of Bulb Temperature

The step responses of the measured quantity $y 3$ (bulb temperature) for the step of $u 1$ (bulb voltage) and constant speed of the main fan $(u 2=2 \mathrm{~V})$ are depicted in Figure 3. It is clear from the figure that the step responses have a nonlinear behaviour. The plant acts as it has a short time constant at the beginning and it slows down at the end of history. Hence it should be taken into account that the time constant will vary in a large range. It follows from the steady-state load characteristic that the gain varies in the range of 0.42 to 0.54 . The family of transfer function from $u 1$ to $y 3$ at $u 2=2 \mathrm{~V}$ is:

$$
\mathbf{P}_{13}=\left\{\frac{k}{T s+1}: k \in(0.42 ; 0.54), T \in(5 ; 20)\right\}
$$

This means that both the numerator and denominator in (1) are interval polynomials.

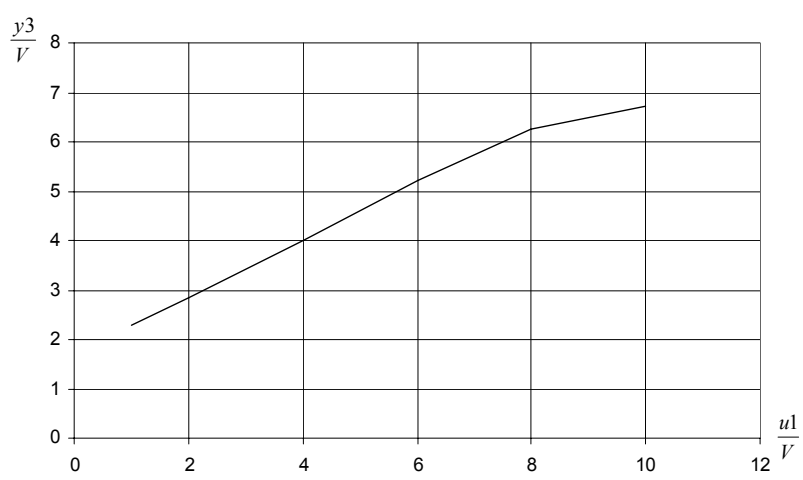

Figure 4: Steady-State Load Characteristic

\section{OUTLINE OF POLE PLACEMENT DESIGN}

The pole placement principle is one of the well-known methods for the controller design (e.g. Kucera 1991, Kucera 1993, Prokop and Corriou 1997, Prokop et al. 1992, Vidyasagar 1985) which is simple for derivation and tuning. Consider a simple feedback loop (1DOF) structure depicted in Figure 5 with two external inputs the reference $w$ and disturbance $v$ respectively. The output and tracking error is according to Figure 5 in the form

$$
\begin{aligned}
& y(s)=\frac{b q}{d} w(s)+\frac{c f p}{d} v(s) \\
& e(s)=w(s)-y(s)=\frac{a f p}{d} \cdot \frac{h_{w}}{f_{w}}-\frac{c f p}{d} \cdot \frac{h_{v}}{f_{v}}
\end{aligned}
$$

where

$$
a f p+b q=d
$$

is the characteristic polynomial of the closed-loop system in Figure 5.
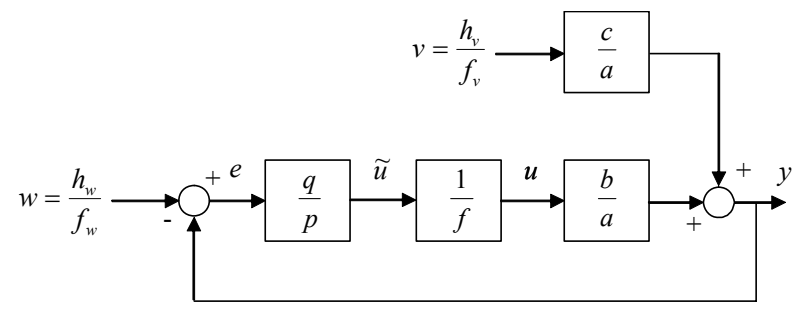

Figure 5: Structure of 1DOF system 
It can be proven that the asymptotic tracking of the reference is achieved if and only if the polynomial $p f a$ is divisible by the unstable part of $f_{w}$ and $v$ is rejected if $p f a$ is divisible by the unstable part of $f_{v}$. As a consequence, the polynomials $p$, $q$ are the solutions to Diophantine equation (4). It is also desirable that the transfer function $\frac{q}{f p}$ is proper. Analysis of the polynomial degrees in (4) for the most frequent case $f_{w}=f=s$ (the stepwise reference) gives

$$
\operatorname{deg} d=2 \operatorname{deg} a
$$

A standard choice for the polynomial $d$ is

$$
d(s)=\prod_{i=1}^{\operatorname{deg} d}\left(s+\alpha_{i}\right) .
$$

where $\alpha_{i}>0$ are the tuning parameters of the controller and $d$ is a stable polynomial which ensures the internal stability of the nominal system.

With respect to (1) a nominal plant transfer function can be expressed by the transfer function

$$
P(s)=\frac{b(s)}{a(s)}=\frac{b_{0}}{s+a_{0}}
$$

and

$$
\begin{aligned}
& w=\frac{h_{w}}{f_{w}}=\frac{1}{s} \\
& v=\frac{h_{v}}{f_{v}}=\frac{1}{s}
\end{aligned}
$$

Then equation (4) has the form

$$
\left(s+a_{0}\right) s+b_{0}\left(q_{1} s+q_{0}\right)=s^{2}+d_{1} s+d_{0}
$$

and by simple equating the coefficients at the like power of $s$ at the left and right of (10) it can be obtained

$$
\begin{aligned}
& q_{1}=\left(d_{1}-a_{0}\right) / b_{0} \\
& q_{0}=d_{0} / b_{0}
\end{aligned}
$$

Then the resulting controller is proper and has the traditional PI structure in the form

$$
Q=\frac{q_{1} s+q_{0}}{s}
$$

\section{PRINCIPLES OF $\mu$-SYNTHESIS}

The parametric uncertainty in $\widetilde{P}_{13}$ can be utilized via the LFT framework by using the additive and quotient uncertainty. Define the nominal plant

$$
P_{13}=\frac{0.48}{12.5 s+1}
$$

The plant family $\widetilde{P}_{13}$ is then equivalent to

$$
\begin{aligned}
& \widetilde{P}_{13}(s, \Delta)=\frac{0.48+0.06 \delta_{2}}{12.5 s+1} \frac{1}{1+\frac{7.5 \delta_{3} s}{12.5 s+1}} \\
& \Delta=\left[\begin{array}{cc}
\delta_{2} & 0 \\
0 & \delta_{3}
\end{array}\right], \delta_{2}, \delta_{3} \in(-1 ;+1)
\end{aligned}
$$

Let

$$
W_{2}(s)=\frac{0.06}{12.5 s+1}, W_{3}(s)=\frac{7.5 s}{12.5 s+1}
$$

then

$$
\widetilde{P}_{13}(s, \Delta)=\left(P_{13}+\delta_{2} W_{2}\right) \frac{1}{1+\delta_{3} W_{3}}
$$

The expression (16) is represented by the LFT interconnection depicted in Figure 6.

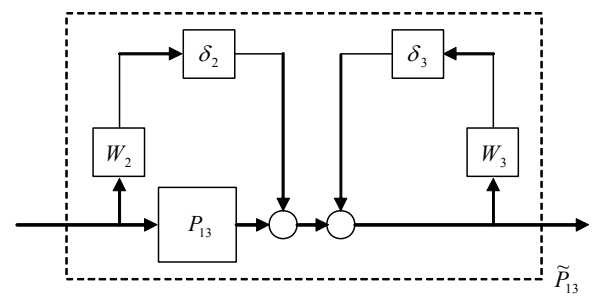

Figure 6: LFT Representation of $\widetilde{P}_{13}$

The LFT interconnection for the $\mu$-synthesis which considers the performance objectives and noise suppression is in Figure 7.

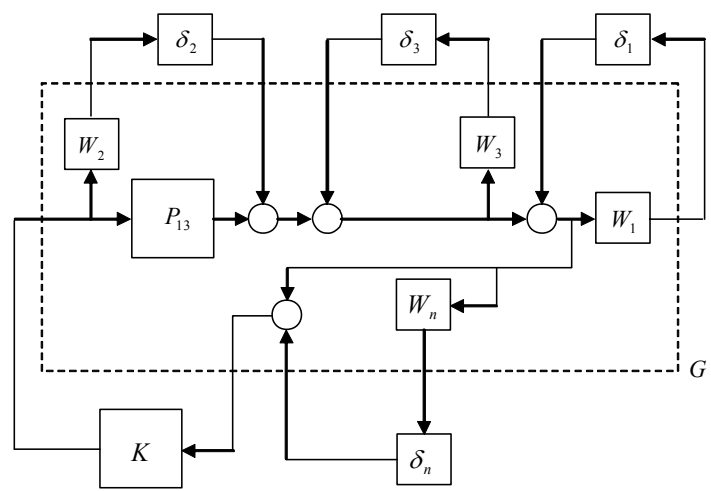

Figure 7: LFT Interconnection for $\mu$-Synthesis

The weight $W_{1}$ for the performance evaluation was chosen as

$$
W_{1}(s)=\frac{0.01 s+1}{s+0.0001} 0.09 \text { and } \frac{0.01 s+1}{s} 0.09
$$


for the $D-K$ iteration and algebraic approach, respectively. The weight for the $D-K$ iteration cannot have integrating behaviour because all weights must be stable. Moreover, it causes uncontrollable states in the closed-loop system. The instability and uncontrollability of the closed-loop in Figure 7 does not make the resulting feedback loop unstable if there is a guarantee that the poles of the nominal feedback loop are in the left half plane. Controllability is a necessary condition for using the state space formulae giving the $\mathbf{H}_{\infty}$ suboptimal controller (Doyle et al. 1989) as well as stability of all weighting functions. Thus it is impossible to use these formulae in this case. The algebraic approach overcomes the problem by setting the nominal closed-loop poles to the left half-plane. Therefore, it is possible to use performance weights with poles at the imaginary axis, which guarantee the asymptotic tracking.

The weight of noise is a band-pass filter, which takes into account high frequency noise emerging in sensors

$$
W_{n}(s)=\frac{1 / 0.001 s+1}{1 / 0.5 s+1} 0.001
$$

Let $S$ denote a perturbed transfer function from the reference input $w$ to the tracking error $e$. Let $W_{1}$ denote the weighting function and define the performance condition as

$$
\left\|W_{1} S\right\|_{\infty} \leq 1
$$

If the condition (19) holds then behaviour of the closed loop can be changed through $W_{1}$.

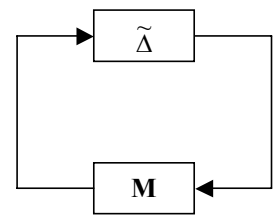

Figure 8: Transformed Closed-Loop System

The closed-loop feedback system in Figure 7 can be transformed to that in Figure 8, where $\mathbf{M}$ is a linear fractional transformation on $\mathbf{G}(s)$ and controller $K(s)$, i.e.,

$$
\mathbf{M}=\mathbf{F}_{l}(\mathbf{G}, K)=\mathbf{G}_{11}+\mathbf{G}_{12} K\left(1-G_{22} K\right)^{-1} \mathbf{G}_{21}
$$

where $\mathbf{G}(s)$ is the generalized plant including the nominal plant and weighting functions, which can be parted to

$$
\mathbf{G}(s)=\left[\begin{array}{ll}
\mathbf{G}_{11} & \mathbf{G}_{12} \\
\mathbf{G}_{21} & G_{22}
\end{array}\right]
$$

where the rows and columns of $\mathbf{G}_{11}$ correspond to dynamic perturbations (22) and $G_{22}$ corresponds to the controller structure. The other element of the system is dynamic structured uncertainty which forms the diagonal matrix

$$
\widetilde{\boldsymbol{\Delta}}=\left\{\operatorname{diag}\left[\delta_{1}, \delta_{2}, \delta_{3}, \delta_{n}\right]: \delta_{2,3} \in \mathbf{R}, \delta_{1, n} \in \mathbf{C}\right\}
$$

The algebraic $\mu$-synthesis is applied to the 1DOF system for the interconnection depicted in Figure 7. The $D-K$ iteration is applied to the same structure with $W_{1}$ without integrating behaviour.

The structured singular value of a matrix $\mathbf{M}$, denoted $\mu_{\tilde{\Delta}}(\mathbf{M})$, is defined as

$$
\mu_{\widetilde{\Delta}}(\mathbf{M})=\frac{1}{\min \{\tilde{\sigma}(\widetilde{\Delta}): \operatorname{det}(I-\mathbf{M} \widetilde{\Delta})=0\}}
$$

and if no such $\widetilde{\Delta}$ exists which makes $\mathbf{I}-\mathbf{M} \widetilde{\Delta}$ singular, let $\mu_{\widetilde{\Delta}}(\mathbf{M})=0$ (Levine 1996), where $\bar{\sigma}(\widetilde{\Delta})$ denotes the maximum singular value. The control objective is to find a stabilizing controller $\mathbf{K}$ minimizing the $\mathbf{H}_{\infty}$ norm of $\mu_{\tilde{\Delta}}(\mathbf{M})$, i.e.,

$$
\min _{\mathbf{K} \text { stabilizing } \mathbf{G}}\left\|\mu_{\tilde{\Delta}}\left[\mathbf{F}_{l}(\mathbf{G}, \mathbf{K})\right]\right\|_{\infty}
$$

The following result is used for the robust performance test (Levine 1996):

The feedback system with $|\widetilde{\boldsymbol{\Delta}}|<1$ has the robust performance, i.e., expression (19) holds and the perturbed feedback loop is internally stable, if and only if

$$
\mu_{\widetilde{\Delta}}(\mathbf{M}) \leq 1
$$

at all frequencies.

In the algebraic approach the nominal closed-loop poles are the tuning parameters and the quality of the controller is evaluated by the upper bound for $\mu$. The poles are constraint to the real axis in the left half-plane so that the stability of the nominal feedback loop is guaranteed. As the cost function defined by (24) can have more than one local minimum an algorithm for global optimization is desirable. In this contribution Differential Migration (Dlapa 2009) was used for the optimization since it yields high efficiency in finding the global minimum. Differential Migration is an evolutionary algorithm based on migration of individuals in the space of tuning parameters giving significantly higher robustness (in the sense of ability of finding the global minimum) than other algorithms of this class. The usage of this algorithm can shorten the computational time and increase the probability of finding the optimal pole placement. 


\section{APPLICATION TO AIR-HEATING PLANT}

Experimental studies have been carried out in order to assess the performance of the algebraic $\mu$-synthesis method. The set-point temperature profile provides a reference which comprises 800 iterations. It consists of an initial soak at $3 \mathrm{~V}$ for 480 iterations followed by a step to $4 \mathrm{~V}$ which is held constant for 300 iterations. Sampling period is $1 s$ and adjacent fan voltage is ketp zero for all experiments.

The experimental trials are aimed at evaluating the performance of the PI controller obtained via the algebraic $\mu$-synthesis against the $D-K$ iteration, synthesis in the $R_{\mathrm{PS}}$ and the Naslin method.

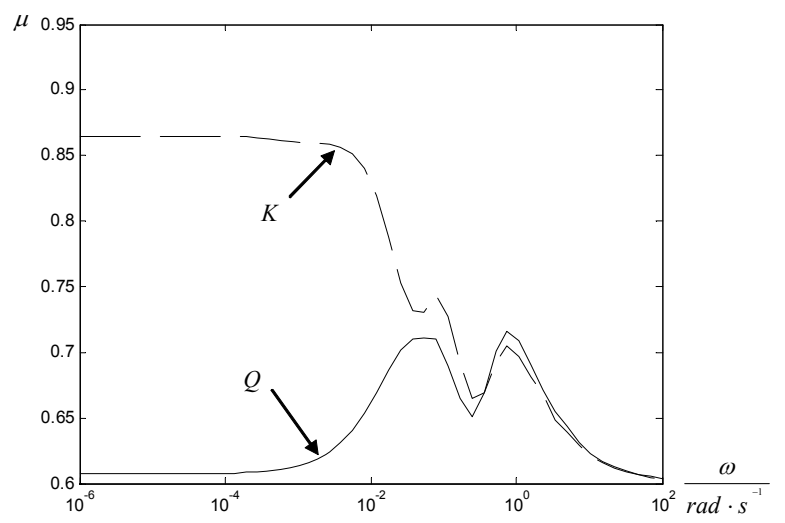

Figure 9: $\mu$-Plot for $D$ - $K$ Iteration (dashed) and Algebraic $\mu$-Synthesis (solid)

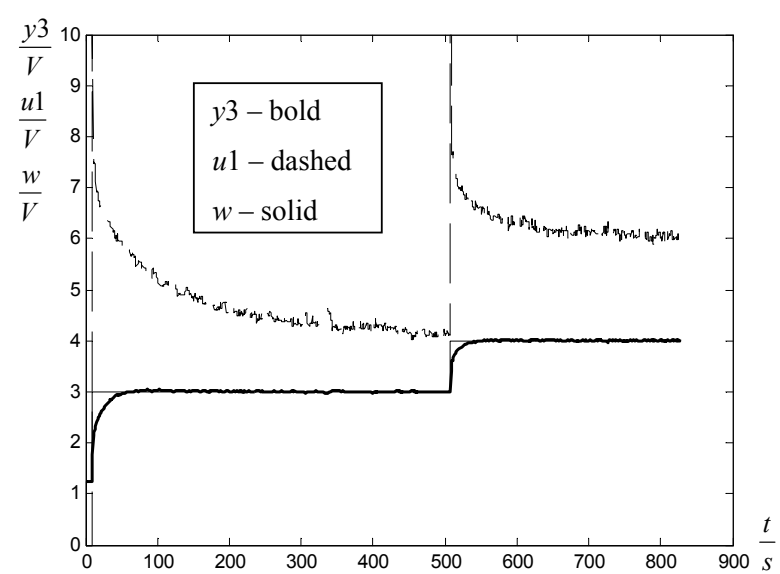

Figure 10: Control of Real Plant for Algebraic Approach

Performance indices. In order to draw comparisons between different control schemes an index or measure of performance is required. The measure of effectiveness which is adopted consists of three factors, these being the amount of energy, the variance of the controlled actuators and the accuracy of set-point tracking. This may be expressed as

$$
\epsilon_{1}=\frac{\sum u(t)}{\rho}
$$

where $\rho$ is the number of iterations.

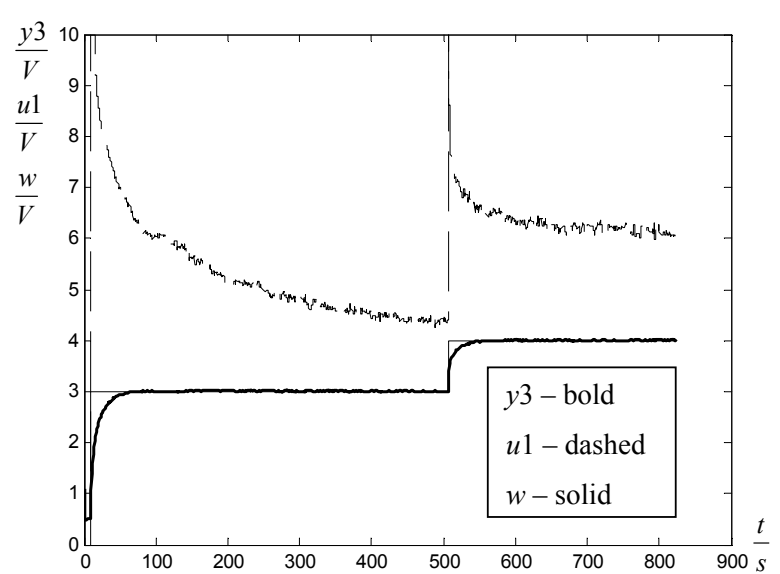

Figure 11: Control of Real Plant for $D-K$ Iteration

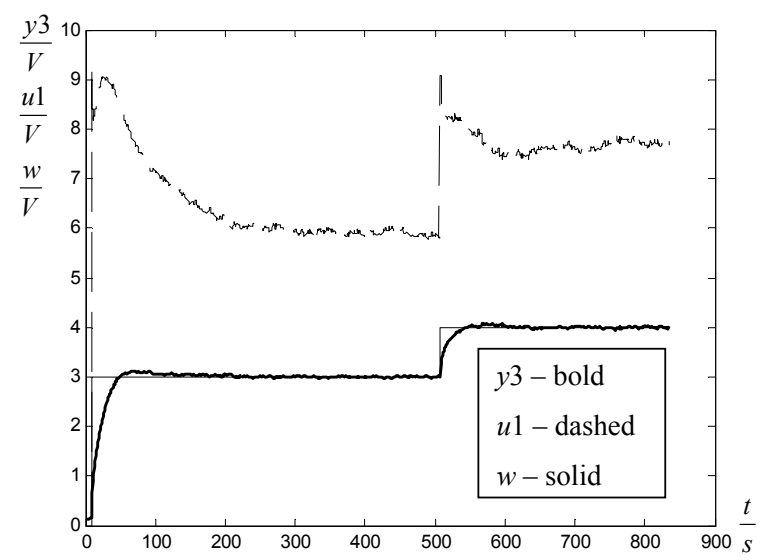

Figure 12: Control of Real Plant for Synthesis in $R_{\mathrm{PS}}$

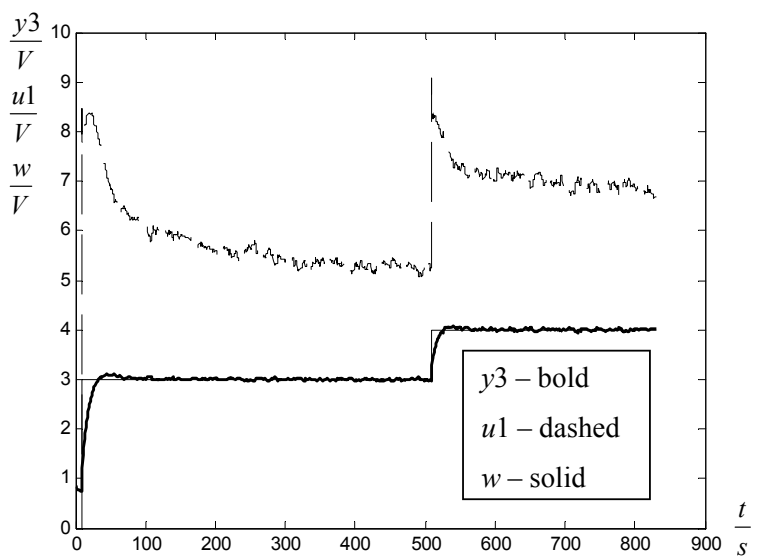

Figure 13: Control of Real Plant for Naslin Method

In cases where there is an increased variance in the control signals to the actuator this can lead to correspondingly increased costs due to maintenance and down time due to failure. The variance of the controlled actuator may be expressed in the form

$$
\epsilon_{2}=\sum\left[u(t)-\epsilon_{1}\right]^{2}
$$

The resulting controller quality arising from control action may be expressed in terms of the accuracy of setpoint tracking. Using the integral of absolute error the 
deviation of the system response $y(t)$ from the set-point $r(t)$ is given as

$$
\epsilon_{3}=\sum|y(t)-r(t)|
$$

In order to provide a basis for comparison the controllers were tuned to give satisfactory overall performance across the complete temperature profile. The controllers designed in the ring of proper and stable functions $\left(R_{\mathrm{PS}}\right.$, see Prokop and Corriou 1997 or Prokop et al. 1992) and by the Naslin method were used as a reference (Figures 12 and 13). It is clear from Figures 9, $10,11,12$, and 13 which show the system response and control input that the algebraic approach produces more favourable results. The algebraic $\mu$-synthesis (Figure 10) achieves an improved accuracy in set-point tracking as well as in reducing the input control effort to the system. The set-point, actuator and measured signals are in the unified voltage $0-10 \mathrm{~V}$ of the CTRL unit. Performance indices are given in Table 2.

Table 2: Comparison of Performance Indices

\begin{tabular}{|c|c|c|c|}
\hline Method & $\epsilon_{1}$ & $\epsilon_{2}$ & $\epsilon_{3}$ \\
\hline Algebraic $\mu$-synthesis & 6.30 & 0.24 & 8.33 \\
\hline$D$ - $K$ iteration & 6.37 & 0.23 & 9.07 \\
\hline$R_{\mathrm{PS}}$ & 7.72 & 0.17 & 13.42 \\
\hline Naslin method & 7.10 & 0.22 & 11.02 \\
\hline
\end{tabular}

\section{CONCLUSION}

The contribution presents an application of the algebraic $\mu$-synthesis to an air-heating set, where the temperature of bulb was controlled by its voltage. The controlled system had a nonlinear behaviour in both the steadystate and dynamic characteristics. The nonlinearity was utilized via parametric uncertainty, which was transformed to the LFT framework. In order to achieve asymptotic tracking, performance weighting function with pole at the imaginary axis was used. The instability of the nominal feedback loop was treated by setting its poles to the left half-plane via pole placement technique and by choosing the PI structure of the controller which treats the unstable pole of the closed loop. The algebraic $\mu$-synthesis was applied to the LFT interconnection including performance weighting function with integrating behaviour and the results were compared with standard methods for robust controller design - the $D-K$ iteration, synthesis in $R_{\mathrm{PS}}$ and the Naslin method. Finally, it was shown that the algebraic $\mu$-synthesis had better frequency properties and set-point tracking than the reference methods.

The algebraic $\mu$-synthesis provides exploitable benefits for a wide range of industrial applications. In contrast to the $D-K$ iteration, it can tune simple controllers in more natural way and guarantee asymptotic tracking, which is desirable in most of the control tasks emerging in technology processes.

\section{ACKNOWLEDGEMENTS}

This work was supported by the Ministry of Education Youth and Sports of the Czech Republic under Grant MSM7088352102 and by the European Regional Development Fund under the Project CEBIA-Tech No. CZ.1.05/2.1.00/03.0089

\section{REFERENCES}

Levine W.S. 1996. The control handbook, CRC Press, Inc., IEEE Press, pp. 671-687.

Doyle J.C. 1982. "Analysis of feedback systems with structured uncertainties," Proceedings of IEE, Part-D, 129, pp. 242-250.

Doyle J.C. 1985. "Structure uncertainty in control system design," Proceedings of 24th IEEE Conference on decision and control, pp. 260-265.

Doyle J.C.; P.P. Khargonekar and B.A. Francis. 1989. State-space solutions to standard $\mathrm{H}_{2}$ and $H_{\infty}$ control problems, IEEE Transactions on Automatic Control, Vol. 34, No. 8, pp. 831-847.

Fiser R. 2002. "Control algorithms of 200 MW production blocks in power plant Chvaletice for stationary and also non-stationary operation, experience, development," Proceedings of Control of power \& heating systems 2002, Zlin, Czech Republic.

Kucera V. 1991. Analysis and design of discrete linear control systems, Academia.

Kucera V. 1993. "Diophantine equations in control - a survey," Automatica, Vol. 29, No. 6, pp. 1361-75.

Lin J. L.; I. Postlethwaite and D. W. Gu. 1993. " $\mu-K$ iteration: a new algorithm for $\mu$ synthesis," Automatica, 29, 219-224.

Liu H.; S. Liand and T. Chai. 2003. "Intelligent decoupling control of power plant main steam pressure and power output," International Journal of Electrical Power \& Energy Systems, Vol. 25, No. 10, pp. 809-819.

Prokop R. and J. P. Corriou. 1997. "Design and analysis of simple robust controllers," Int. J. Control, Vol. 66, No. 6, pp 905-921.

Prokop R.; P. Dostal and A. Meszaros. 1992. "Continuous-time control via proper regulators," Journal A, Vol. 33, No. 4.

Vidyasagar M. 1985. Control system synthesis - A factorization approach, MIT Press Cambridge.

Zames G. 1981. "Feedback and optimal sensitivity: model reference transformations, multiplicative seminorms, and approximate inverse," IEEE Trans. Auto. Control, 26, pp. 301-320.

Dlapa, M. 2009. "Differential Migration: Sensitivity Analysis and Comparison Study," Proceedings of 2009 IEEE Congress on Evolutionary Computation (IEEE CEC 2009), pp. 1729-1736, ISBN 978-1-4244-2959-2.

Dlapa, M.; R. Prokop and M. Bakošová. 2009. "Robust Control of a Two Tank System Using Algebraic Approach," Proceedings of EUROCAST 2009, pp. 603-609, ISBN 978-84-691-8502-5.

Dlapa, M. and R., Prokop. 2010. Algebraic approach to controller design using structured singular value, Control Engineering Practice, Vol. 18, No. 4, pp. 358-382, ISSN 0967-0661. 


\section{AUTHOR BIOGRAPHIES}

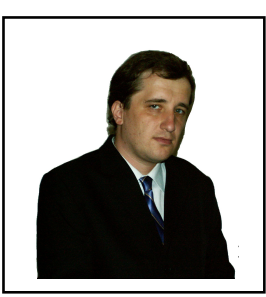

MAREK DLAPA was born in Zlín, Czech Rep. and went to the Tomas Bata University in Zlín, where he studied chemical technology and obtained his degree in 2000. After a couple of years he was given Ph.D. degree at TBU in Zlín in 2004. Now he works as a research worker at TBU in Zlín. His e-mail address is: dlapa@fai.utb.cz and his Web page can be found at http://dlapa.wz.cz. 